\title{
EFFECT OF FRACTURE SURFACE INTERFERENCE ON SHEAR CRACK GROWTH
}

Progress Report

for Period September 1, 1990 to April 30, 1992

DOE/ER' $45433--1$

Todd S. Gross and David W. Watt

Department of Mechanical Engineering

DE92 011431

University of New Hampshire

Durham, NH 03824-3591

and

Daniel A. Mendelsohn

Department of Engineering Mechanics

Ohio State University

Columbus, $\mathrm{OH} 43210$

February 1992

Prepared for

THE U.S. DEPARTMENT OF ENERGY

AGREEMENT NO. DE-FG02-90ER45433.A000

\section{DISCLAIMER}

This report was prepared as an account of work sponsored by an agency of the United States Government. Neither the United States Government nor any agency thereof, nor any of their employees, makes any warranty, express or implied, or assumes any legal liability or responsibility for the accuracy, completeness, or usefulness of any information, apparatus, product, or process disclosed, or represents that its use would not infringe privately owned rights. Reference herein to any specific commercial product, process, or service by trade name, trademark, manufacturer, or otherwise does not necessarily constitute or imply its endorsement, recommendation, or favoring by the United States Government or any agency thereof. The views and opinions of authors expressed herein do not necessarily state or reflect those of the United States Government or any agency thereof.

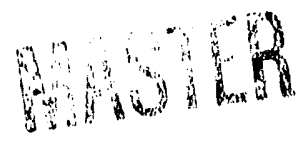


Table of Contents

1. Project Summary

2. Accomplishments at UNH

a. Fracture Surface Profile Characterization

b. Electro - optic Holographic Interferometry

c. Modification of Servohydraulic Testing Frame for Vibration-free Operation

3. Accomplishments at OSU

a. Boundary Element Formulation

b. Computer code development

4. Proposed Work for Remainder of Current Funding Period
a. UNH Effort
b. OSU Effort

5. Proposed Work for Mily 1, 1992 - February 28, 1993 Funding Period
a. UNH Effor"
b. OSU Effort

6. Students Funded by This Project

7. Publications

8. Other Federal Funding 


\section{Project Summary}

A joint analytical -experimental program to investigate the effect of fracture surface interference on shear modes of crack growth is progressing satisfactorily.

A general two-dimensional, boundary element model has been formulated by the group at Ohio State University that is capable of calculating the effective Mode I and Mode II stress intensity factors for flat or curved cracks with small scale yielding. The model can calculate $\mathrm{K}_{\mathrm{I}}$ and $\mathrm{K}_{\Pi}$ for an arbitrary constitutive law for displacement of the crack faces. The constitutive law proposed in our earlier work is being used in the new boundary element model. The difference is that previously, the crack face shear displacements were assumed to follow a $\mathrm{r}^{1 / 2}$ dependence, where no such assumptions are necessary in the boundary element model.

The experimental portion of the effort at UNH was to use an as-yet undeveloped electro-optic holographic interferometry (EOH) system to measure the crack face displacement field while the crack is loaded in shear. The algorithms for obtaining the interferograms have been developed, the testing machine modifications necessary for interferometric measurements are complete, and interferograms of specimens under load have been obtained. Techniques for digitizing the fracture surface profile have been developed and preliminary numerical experiments have been conducted to determine the $\Delta \mathrm{K}$ dependence of fracture asperity interference on an actual crack. 


\section{Accomplishments at UNH}

\section{2.a. Fracture Surface Profile Characterization}

Fourier transform techniques were developed to characterize the amplitude and wavelength of the mode I fatigue fracture surface profile in several different materials. It was proposed that the dominant amplitude and wavelength would be used as input to the boundary element model of the mode II crack and to help analyze the load-displacement maps obtained when the specimen was loaded in mode II. The tasks for this portion of the effort were;

1. Develop metallographic preparation techniques to preserve the fracture surface proille edge.

2. Develop techniques to digitize the fracture surface profile for Fourier transformation.

3. Write a program to obtain the spectrum of amplitudes and wavelengths from the digitized fracture surface profile.

Retention of the fracture surface profile edge is necessary for accurate digitization. The standard method for edge preservation is electroplating with some metal. A 4140 annealed steel fracture surface and an $6061-\mathrm{T} 6$ aluminum fracture surface were successfully plated with $\mathrm{Ni}$. However, we were unable to obtain a continuous coating on a $\mathrm{Ti}-6 \mathrm{Al}-4 \mathrm{~V}$ fracture surface. It was determined that standard polishing of a specimen which had an intact crack tip was sufficient to preserve the edge for digitization. This method will be used to characterize the mode I fatigue crack in each specimen before it is loaded in mode II. We will grind off the mode I plane stress region on both sides of the specimen before loading in mode II.

Two computer programs were developed to detect the fracture surface edge from the digitized intensity on the video image. In one approach, a Robert's edge detection algorithm, included in the frame grabber software, was used with qualified success. In the second approach, the position of the edge was defined as maximum intensity change on a scan perpendicular to the profile. Both approaches required some user intervention to fill in gaps or to correct obviously incorrect points.

A program to compute the Fourier transform of the fracture surface profile was adapted from Numerical Recipes in C. The digitized data was the input and the output was plotted as amplitude vs. wavelength. Video image frames of four, $400 \mathrm{X}$ overlapping micrographs of a 4140 steel fatigue fracture surface profile were digitized. The digitized and transformed protile is shown in in Fig. $1 \mathrm{a}$ and $\mathrm{b}$. The maximum amplitude frequency component is superimposed over the digitized profile. It is clear that selecting a single waveform is not an appropriate description of the how the actual crack will open up during shear loading. As the crack faces are sheared past one another, they must onen so that the crack faces do not occupy the same volume. Figure $2 b$ is the required COD for the actual profile in Fig. la assuming theoretical mode Il crack face displacements with crack tip plasticity (Fig 2a). The imposed COD is significantly different than that predicted from the single waveform in Fig la. We are currently discussing ways to incorporate all of the information from the digitized fracture profile to form the constitutive law for the crack faces. 


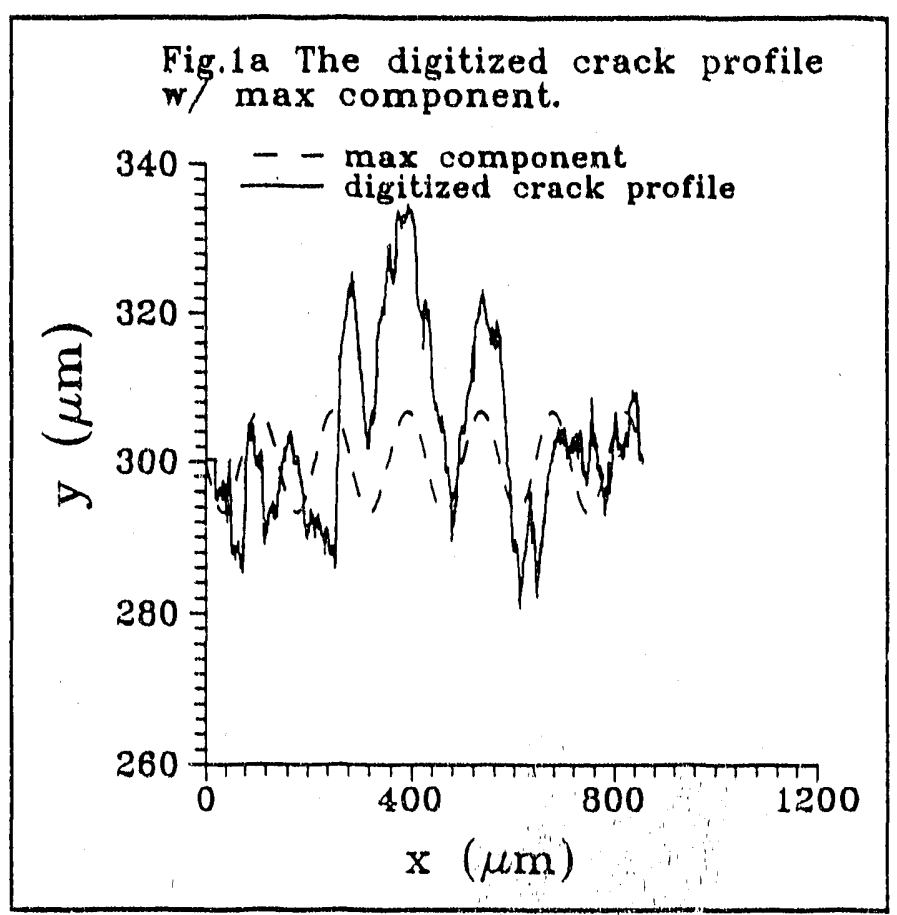

Figure 1a - Digitized fracture surface" profile with superimposed dominant amplitude and wavelength.

Fig.1b The transformed spectrum of the digitized crack profile.

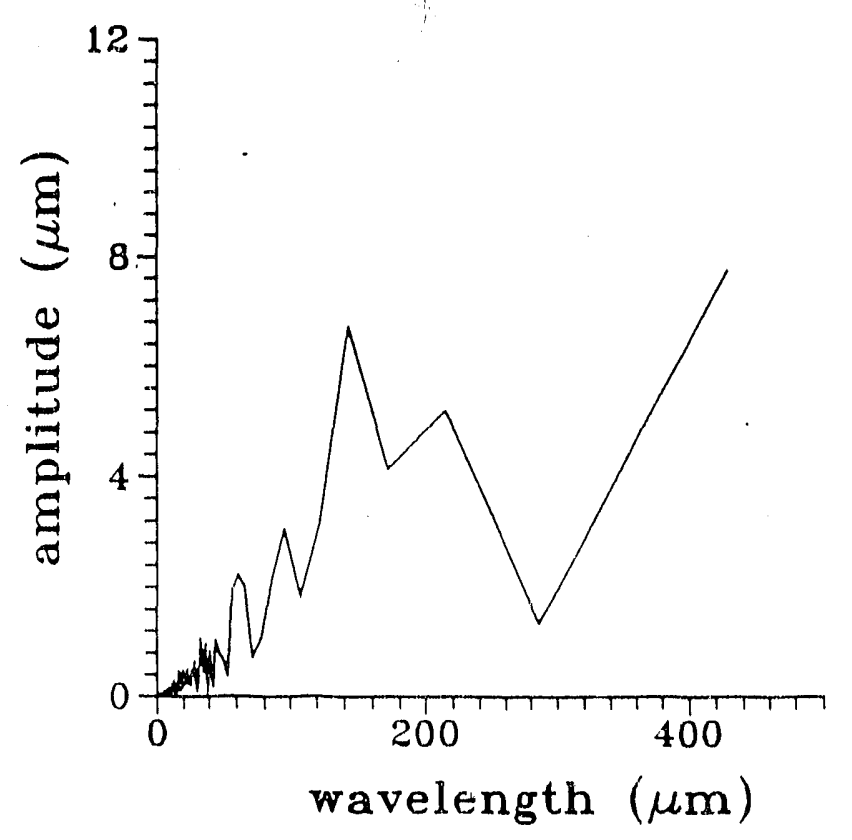

Figure 1b - Amplitude vs wavelength obtained from Fourier transform of digitized fracture surface profile 


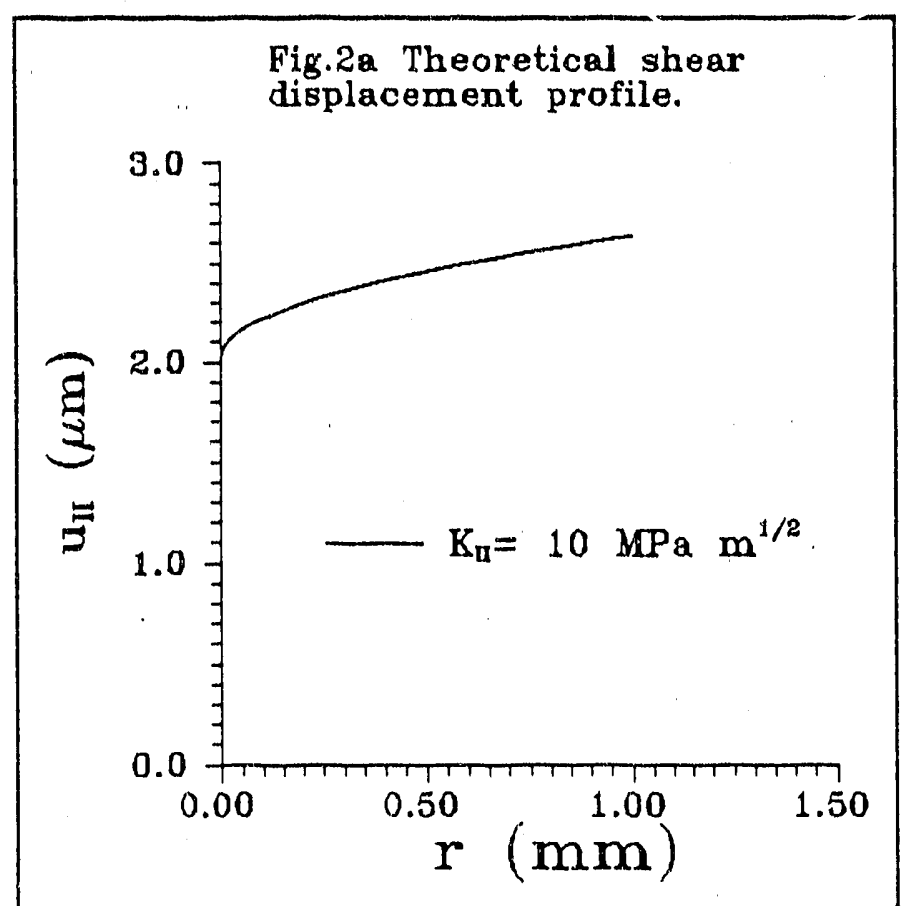

Figure 2a - Crack face displacements with crack tip plasticity.

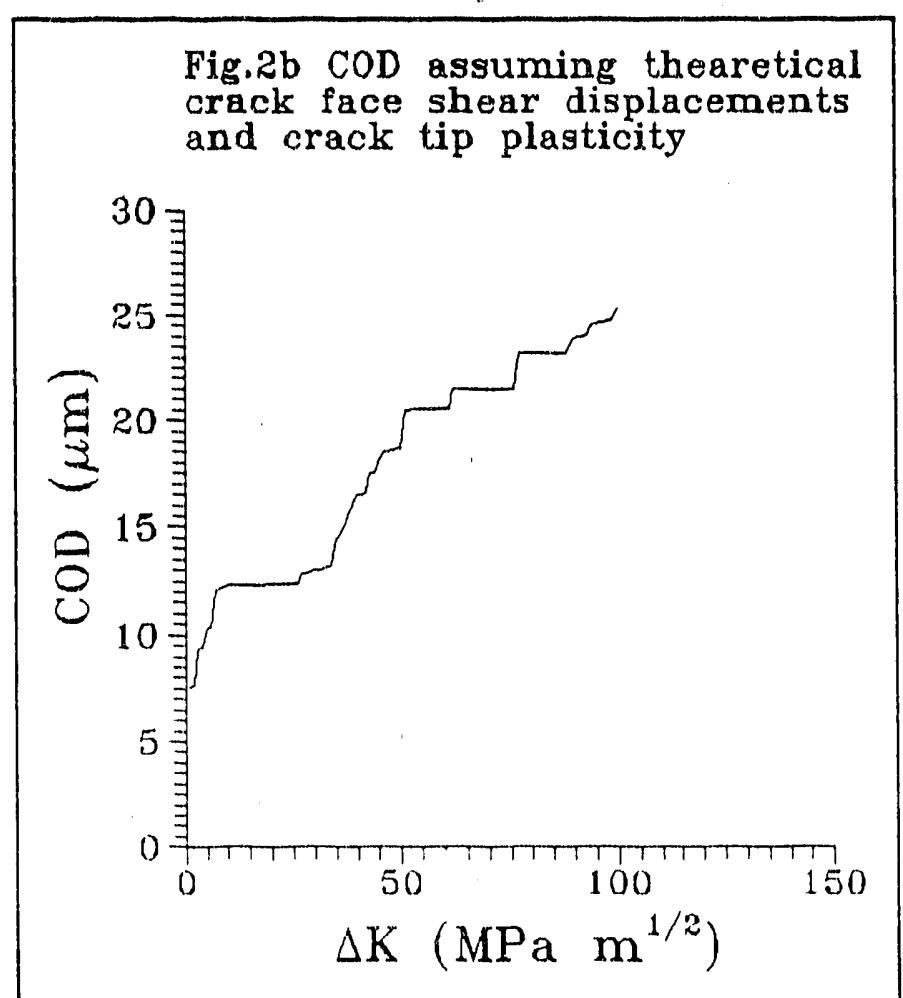

Figure $2 b$ - Induced crack opening displacements for crack face shear displacements on crack profile in Fig. 1. 


\section{2.b. Electro-optic Holographic Interferometry}

The success of the experimental portion of the project depended on the ability to obtain high spatial resolution interferograms utilizing an as - yet developed electro-optic holographic interferometry (EOHI) system. We have developed the software and hardware to obtain successful interferograms with support frcm this and other projects. Scott Raber, an M.S. candidate partially supported by this project, recently defended his thesis dissertation entitled, "Electro-optic Interferometry with Noise Tolerant Phase Unwrapping". The EOHI system at UNH is unique in that it has high spatial resolution enabled by noise reduction from median filtering of the phase images. Descriptions of EOHI systems have only been reported in the literature in the past five years. The bulk of those reports focused on fringe visualization and not phase determination necessary for displacement measurement. Most other investigators deal with the impulsive noise in the phase calculation by averaging over a fairly large nuruber of pixeis (picture elements). The current displacement sensitivity is $\pm 10 \mathrm{~nm}$ for out-of-plane displacements. We have not yet obtained a set of interferograms necessary to obtain the in-plane displacement sensitivity.

\section{2.c. Modification of Servohydraulic Test Frame for}

Vibration Free Operation

The interferometric measurements require that the object be relatively vibration - free since the anticipated displacement accuracy is in the $10 \mathrm{~nm}$ range. Therefore, it was necessary to modify the servohydraulic test frame to run in a vibration - free test mode. The major source of vibration in a servohydraulic testing machine is the electric motor used to maintain 3000 psi pressure in the hydraulic actuator. While the motor must constantly run for fatigue applications, the proposed measurements did not require high volume flow of oil through the actuator. Accordingly, the hydraulic system of a model 1350 Instron machine were modified to run on accumulated pressure in a 5 gallon pressure accumulator. The system was set up so that the electric motor would stop when the pressure reached $3000 \mathrm{psi}$ in the actuator and the accumulator. The electric motor was set to restart and charge the accumulator when the pressure dropped to $1800 \mathrm{psi}$. This allowed approximately $3-5$ minutes of "silent' operation to make the interferometric measurements at a static load. The maximum load was reduced from $20,000 \mathrm{lbs}$ to $12,000 \mathrm{lbs}$ since the maximum load capacity is linearly proportional to the hydraulic pressure. This is not a limiting problem because most of the anticipated specimens will require loads in the $2000 \mathrm{lb}$ range. A significant amount of time was spent to make sure that all of the interlocks were still functional after the modifications to ensure safe operation of the machine. Engineers from Instron were extremely helpful in the redesign of the hydraulic plumbing and interlocks. This modification was an extremely time consuming effort.

We have obtained high quality interferograms from a mode I crack growth specimen and from a specimen with a circilar hole loaded in compression as evidence that the above modifications were sufficient to overeome the vibration problem. However, we have not yet been able to obtain interferograms from three separate illumination beams (necessary to compute the three dimensional displacement field from the scalar phase measurement) at constant load. This is related to beam-specimen alignment problems which result in unequal reflected intensity 
from the different beams. Since we have already demonstrated the capability to back out the three dimensional displacement field on a heated circuit board and on a wedge loaded miniature mode I fatigue crack specimen, the current inability to obtain interferograms using all three beams is a minor problem that will be overcome by more careful alignment. As far as we know, the electro-optic holographic interferometry system mounted on an active servohydraulic frame is the only such system in this country.

\section{Accomplishments at OSU}

\section{3.a. Boundary Element Formulations}

The main features of the boundary element formulation to date are presented first, followed by a list of compieted tasks. The analysis is $2 \mathrm{D}$, plane strain and calculates $\mathrm{K}_{\mathrm{I}}$ and $\mathrm{K}_{\mathrm{II}}$ and crack opening and sliding displacements of a microscopically rough crack subjected to arbitrary loading. The macroscopic crack surfaces need not be planar, e.g. curved cracks are included. The crack may be in a homogeneous elastic material or at the interface between two dissimilar elastic materials.

The crack surface is modelled by a uniform sawtooth distribution of rigid asperities, which when perfectly mated result in zero relative mode I or mode II displacement (gap, or shift, respectively) of the opposing macro crack planes. When the asperity surfaces are in contact the relative motion of the macro crack planes is governed by the geometry of the rigid asperities and by Coulomb frictional stick-slip behavior on the active contact plane. Also, at a critical absolute value of the shift at a given point, it is assumed that opposing asperities. wear to flat planes parallel to the original macro crack plane while maintaining the gap produced by the critical shear displacement. Thus the model requires four independent microstructural parameters: the asperity slope and coefficient of fraction, either the unworn asperity height or the asperity wavelength, and either the worn asperity height or the critical shift for wear.

For intact asperities the active contact plane is the left or right inclined asperity surface (depending on the sign of the shift) and after wear it is the flat worn tops of the asperities. Thus, before or after weal: even though the actual crack surfaces are in partial contact, the macro crack planes are held apart inducing a positive opening mode $\mathrm{K}_{\mathrm{I}}$ in addition to that due to superposed mode I loading. Crack opening sufficient to pull the asperities out of contact entirely at some point or points on the crack can only be caused by superimposed mode I loading. In this case the crack boundary conditions (BC's) revert to the traditional stress free BC's.

The analysis is non-linear and requires iteration to delineate the sizes and locations of the open, stick, slip. wear and crack tip plastic zones along the crack plane at each increment. For non-monotonic loading the stickslip problem is history dependent and requires an incremental formulation with iteration at each increment. Besides $B C ' s$, in each of the crack zones (contact, stick, slip, wear and yield) certain inequality constraints must be satisfied. 


\section{Completed Analytical Tasks}

- The $\mathrm{BC}^{\prime} \mathrm{s}$ and constraints for points on opposing crack surfaces for all of the model crack conditions discussed above have been developed, including coordinete transformations for arbitrarily oriented cracks. Traction and/or displacement BC's on external boundaries are allowed for.

- Small scale yielding BC's ahead of the actual crack tip have been developed for both Irwin and Dugdale models.

- The algebraic reduction of the boundary nodal unknowns (two displacements and iwo tractions per node) via the BC's to obtain a linear system of equations in the remaining unknowns (two per node) has been completed.

- Flow charts for the routines controlling the iteration via the governing inequality constraints have been written.

\section{3.b. Computer Code Development}

The boundary element method requires discretization of the two opposing macro crack planes and a portion of the ligament ahead of the crack, as well as any external boundaries, e.g. the outer edges of a compact tension specimen, into a mesh with $\mathrm{N}$ nodes and surrounding elements. The governing energy balance boundary integral equation, when written over the ciiscretized boundary (mesh), yields a set of algebraic equations in $2 \mathrm{~N}$ unknowns. At each node the unknowns are two of the four nodal boundary displacement and traction components. Which two depends on the BC's at that node. The coefficients of the unknowns in the algebraic system are integrals of the elastic Greesi's functions over the boundary elements. The right hand sides of the equations are the known elastic displacements due to the applied loading in the absence of the crack or any finite boundaries.

\section{Completed Code Tasks}

- A mesh generation routine has been written and tested.

- The coefficients of the algebraic system were integrated in closed form, coded and tested separately for each type of integral. This extra step in the analysis, vs. numerical integration, will save significant computer time.

- The main program which assembles the coefficient matrix and right hand side vector has been written and tested for two simple sets of crack BC's.

- Results for Mode I loading of the traditional stress - free crack have been compared successfully to known $\mathrm{K}_{\mathrm{I}}$ values.

- The results for the rough unworn crack with sufficient applied Mode II loading to prevent frictional locking everywhere along the crack are reasonable and a parameter study is in progress.

- The treatment of external boundaries has been tested by using the geometry of an edge crack in a compact tension specimen for the calculators. 


\section{Proposed Work for Remainder of Current Funding Period}

\section{4.a. UNH Effort}

The bulk of the UNH effort to this date has been the design and construction of the electro-optic holographic interferometry system, development of techniques to characterize the fracture surface profile, and modification of the servohydraulic system. The remainder of the period will be devoted to:

1. Design and fabricate the grips for mode II loading.

2. Finish the installation of shutters, etc in the beam illumination system.

3. Obtain interferograms from three independent illumination beams and calculate the three dimensional displacement field.

4. Obtain samples of an ingot metallurgy and a powder metallurgy 7000 series Al alloy for initial testing.

We have also obtained approval for two to three sessions on Shear and Mixed Modes of Crack Growth to be held at the 1993 TMS - AIME meeting in Denver. We will continue the program development during the remainder of this period and during the next funding period.

\section{4.b. OSU Effort}

Using the rough, all -slip dilantant BC's in I.B. conduct a complete study of the effects of asperity slope and coefficient of friction, superposed mode I loading, and crack length on $-\mathrm{i}-$ the critical mode II loading for slip, - $\mathrm{ii}$ - the magnitudes of the reduction in $\mathrm{K}_{\mathrm{II}}$ compared to the smooth crack, and - iii - the induced $\mathrm{K}_{\mathrm{I}}$. Near tip stress fields will be calculated and the direction of subsequent crack growth will be predicted. This entire study will be the subject of the first paper to focus on the modelling effort.

\section{5.a. Plan for Funding Period -}

\section{May 1, 1992 - March 1, 1993}

\section{UNA Effort}

By the beginning of this funding period, the EOHI system should be fully functional. The measurement of the crack displacement field will be the focus of our. effort. First, we will study the ingot and powder metallurgy 7000 series $\mathrm{Al}$ alloys to make our initial comparison between fracture surface profiles with high and low amplitude roughness. Both of these alloys will possess relatively low yield strength. The comparison of high and low amplitude roughness in a higher yield strength system will be accomplished with a $\beta$-annealed $\mathrm{Ti}-6 \mathrm{Al}-4 \mathrm{~V}$ alloy. The fatigue cracks in this system are tortuous for low $\Delta \mathrm{K}$ and relatively flat for high $\Delta \mathrm{K}$. Ms. Yongchun Zhang will complete her M.S. thesis during this period and continue her graduate studies at some other university. We will fund another Master's student at this point. 


\section{5.b. Plan for Funding Period .}

May 1, 1992 - March 1, 1993

OSU Effort

- Complete the code development to include all of the features of the asperity model and the non-monotonic loading discussed in 3.a.

- Formulate contact and interference BC's which represent either deterministically (fractals) or statistically the actual fracture surfaces characterized in the laboratory and include them in the boundary element formulation. Several levels of sophistication will be tried starting with point contact only at the observed high point(s) of the surface. Recent work by the PI and colleagues at OSU with statistical descriptions of interfaces for wave scattering problems will be investigated for use in this effort.

- Develop a boundary element model for the specimen geometry and loading to be used in the experiments, and compute the actual stress field which the crack sees.

Investigate the bi-material interface problem.

- Develop a crack - tip element for the mixed mode small scale yielding BC's in 3.a.

6. Students Funded by Project.

1. Mr. R. Scott Raber, M.S. Thesis entitled, "Electro-optic Interferometry with Noise Tolerant Phase Unwrapping" December 1991, UNH

2. Ms. Yongchun Zhang, M.S. Candidate, anticipated fiulish date, August 1992, UNH

3. Mr. Jeffrey Lih-Jer Young, Expected Ph.D. Graduation - Autumn 1993, OSU

\section{Publications Resulting from the Project}

Thermal Strain Response of Plated Holes, T.S. Gross, D.W. Watt, R.S. Raber, J.A. Perault, Y. Zhang, Proceeding of International Electronic Packaging Society, Fall 1991, p. 685-694

\section{Other Federal Funding}

None for UNH or OSU 

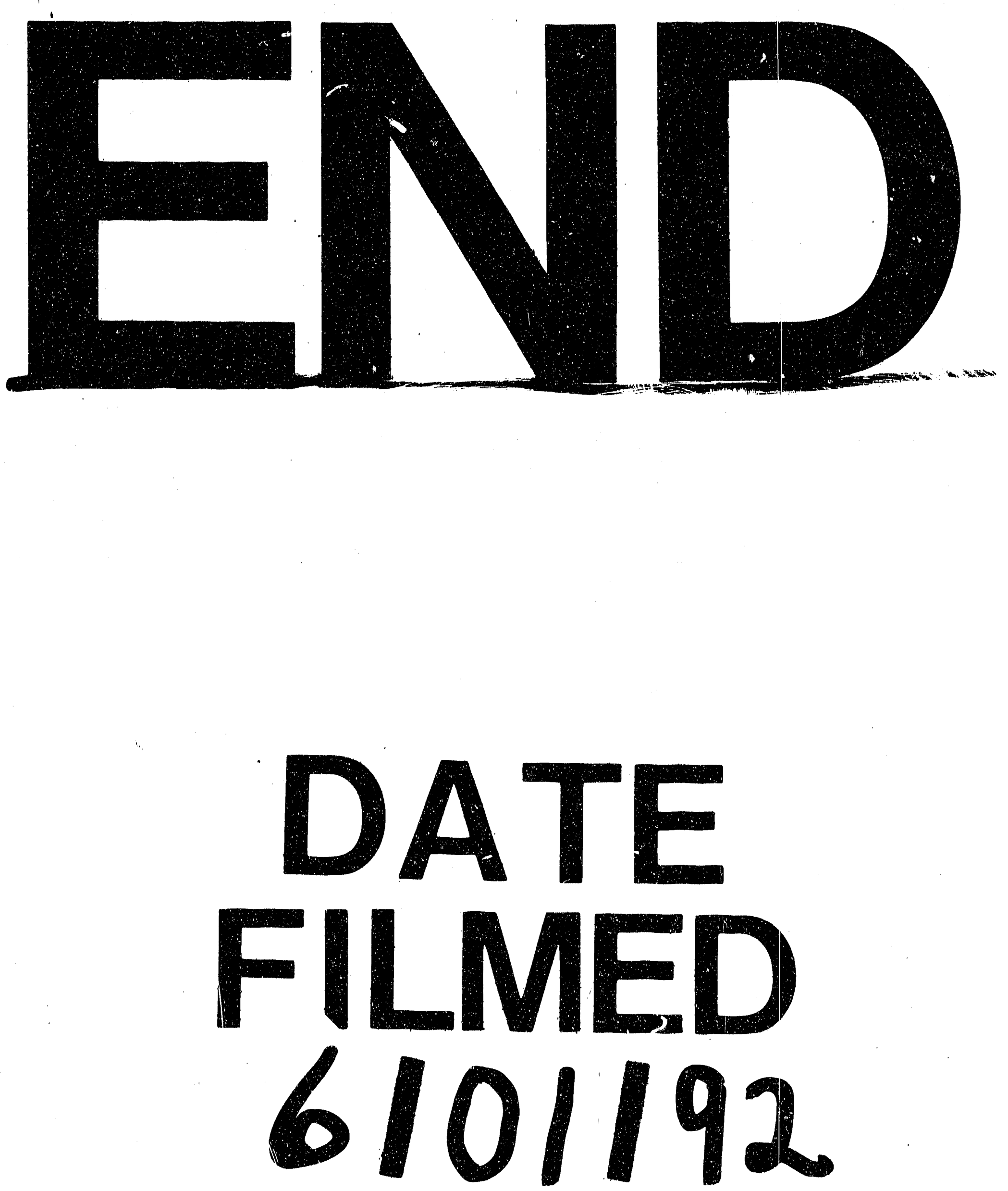